\title{
Fentanyl Citrate
}

National Cancer Institute

\section{Source}

National Cancer Institute. Fentanyl Citrate. NCI Thesaurus. Code C47994.

The citrate salt of fentanyl, a synthetic opioid related to the phenylpiperidines with analgesic and anesthetic properties. Fentanyl exerts its analgesic effect by selectively binding to the mu-opioid receptor in the central nervous system (CNS), thereby mimicking the effects of endogenous opiates. Additional pharmacological effects of fentanyl include anxiolysis, euphoria, feelings of relaxation, respiratory depression, constipation, miosis, and cough suppression. 